\title{
El ecobarrio, una alternativa para el mejoramiento urbano de los asentamientos irregulares ${ }^{1}$
}

\section{Eco-neighborhoods, an alternative for the improvement of illegal settlements}

\author{
María de Lourdes Flores-Lucero*
}

\begin{abstract}
Eco-neighborhoods appear as an alternative to accomplish the principles of sustainable development in degraded urban areas. In Mexico some projects are beginning to be classified as such, but they remain short-sighted and technocratic in their approach. Our goal is to show that the community of San Juan Tulcingo, Puebla, represents an opportunity to transform an illegal settlement into an eco-neighborhood, because despite its urban marginality, it presents sustainable features such as social cohesion, agricultural knowledge, and some habits of non-motorized transportation. Our study is based on four methodological elements: literature review, semi-structured interviews, workshops with residents and field observation.
\end{abstract}

Keywords: illegal settlement, eco-neighborhoods, sustainable urban development, San Juan Tulcingo.

\begin{abstract}
Resumen
Los ecobarrios son una alternativa para materializar los principios del desarrollo sustentable principalmente en zonas urbanas degradadas. En México algunos proyectos empiezan a tener estas características pero con una visión corta y tecnocrática. El objetivo de esta investigación es mostrar que el asentamiento irregular de San Juan Tulcingo, Puebla, puede ser transformado en ecobarrio ya que, a pesar de su marginalidad urbana, presenta rasgos de sustentabilidad como la cohesión social, conocimientos agrícolas, y algunos hábitos de desplazamientos no motorizados. Nuestro estudio se basa en cuatro elementos metodológicos: revisión bibliográfica, entrevistas semidirigidas, talleres con los habitantes y observación en campo.
\end{abstract}

Palabras clave: asentamiento irregular, ecobarrio, desarrollo urbano sustentable, San Juan Tulcingo.

* Benemérita Universidad Autónoma de Puebla. Correo-e: lulu.lucero@gmail.com

${ }^{1}$ Este análisis presenta algunos resultados de la investigación titulada "Mejoramiento y desarrollo urbano del asentamiento San Juan Tulcingo, Puebla: la Ecolonia como proyecto alternativo" Clave FLlm-Ing10-I, VIEP, cuya responsabilidad recae en la autora del presente artículo y en el que participaron como becarios los alumnos Diana Rivera Barragán, Moisés Ernesto Sánchez Castro, María del Carmen Vidal Guridi e Isaac Martínez Javier del Colegio de Urbanismo de la FA-BUAP, y María Isabel Rosas Villanueva de la maestría en ordenamiento del territorio de la FA-BUAP. 


\section{Introducción ${ }^{2}$}

San Juan Tulcingo (sјт) es un asentamiento irregular que actualmente tiene una población de aproximadamente 400 habitantes; aunque cuenta con títulos de propiedad privada es considerado irregular porque no cumple con la normativa urbana. ${ }^{3}$ Se ubica al nororiente de la Zona Metropolitana de la Ciudad de Puebla (ZMCP), en la cuenca alta del río Atoyac, cerca de las faldas de la montańa de la Malinche. Su superficie aproximada es de 27 hectáreas ${ }^{4}$ y empezó su proceso de ocupación hace aproximadamente 20 años.

Según nuestras entrevistas, la poca ocupación del asentamiento se debe, principalmente, a que los terrenos son de propiedad privada y los dueños no habían querido venderlos porque, la mayoría, pensaban lotificarlos y darlos a sus familias. Sin embargo, algunos ya empezaron a ponerlos en venta. Durante el proceso de poblamiento se ha respetado la traza de los terrenos agrícolas, mismos que han sido lotificados por sus propietarios; la mayor parte de las construcciones se concentran en la calle Francisco I. Madero debido a que ésta se localiza a menos distancia de la carretera federal Puebla-Tlaxcala - principal vía de comunicación con la ciudad de Puebla.

El hecho de que un tramo del proyecto del anillo periférico pase a pocos metros del límite sur del asentamiento ha dado inicio a la especulación de los terrenos por parte de los propietarios y al temor de que sus tierras sean expropiadas (figura I). De acuerdo con las entrevistas realizadas, para finales del 2011 estaba previsto el inicio de obras, sin embargo, aún no han empezado. Desgraciadamente este proyecto no está acompańado de un programa de reordenamiento territorial de toda la zona que afecta.

\footnotetext{
${ }^{2}$ Este análisis presenta algunos resultados de la investigación titulada "Mejoramiento y desarrollo urbano del asentamiento San Juan Tulcingo, Puebla: la Ecolonia como proyecto alternativo" Clave FLLM-Ing10-I, viEP, cuya responsabilidad recae en la autora del presente artículo y en el que participaron como becarios los alumnos Diana Rivera Barragán, Moisés Ernesto Sánchez Castro, María del Carmen Vidal Guridi e Isaac Martínez Javier del Colegio de Urbanismo de la FA-BUAP y María Isabel Rosas Villanueva de la maestría en Ordenamiento del Territorio de la FA-BUAP.

${ }^{3}$ De acuerdo con Azuela (1999) son dos las cuestiones jurídicas que se observan en los asentamientos irregulares: por un lado surgen casi siempre al margen del régimen de la planeación, es decir, al conjunto de normas jurídicas mediante las cuales se pretende regular la conformación del espacio urbano desde los aparatos estatales; y por el otro, se origina la llamada tenencia irregular del suelo que se define como la situación en la que los habitantes de un barrio ejercen la posesión pero no la propiedad. En el caso de San Juan Tulcingo la irregularidad se refiere a la primera cuestión marcada por Azuela, pues el único servicio urbano con el que cuenta es la red eléctrica y está sobre zonas destinadas a uso agrí́cola, es decir, no es compatible con el uso de suelo marcado por la carta urbana.

${ }^{4} \mathrm{Si}$ tomamos en cuenta el número de habitantes tendremos una densidad de aproximadamente $15 \mathrm{hab} / \mathrm{ha}$.
} 


\section{Figura I \\ Ubicación de San Juan Tulcingo y su cercanía al proyecto del Anillo periférico ${ }^{5}$}

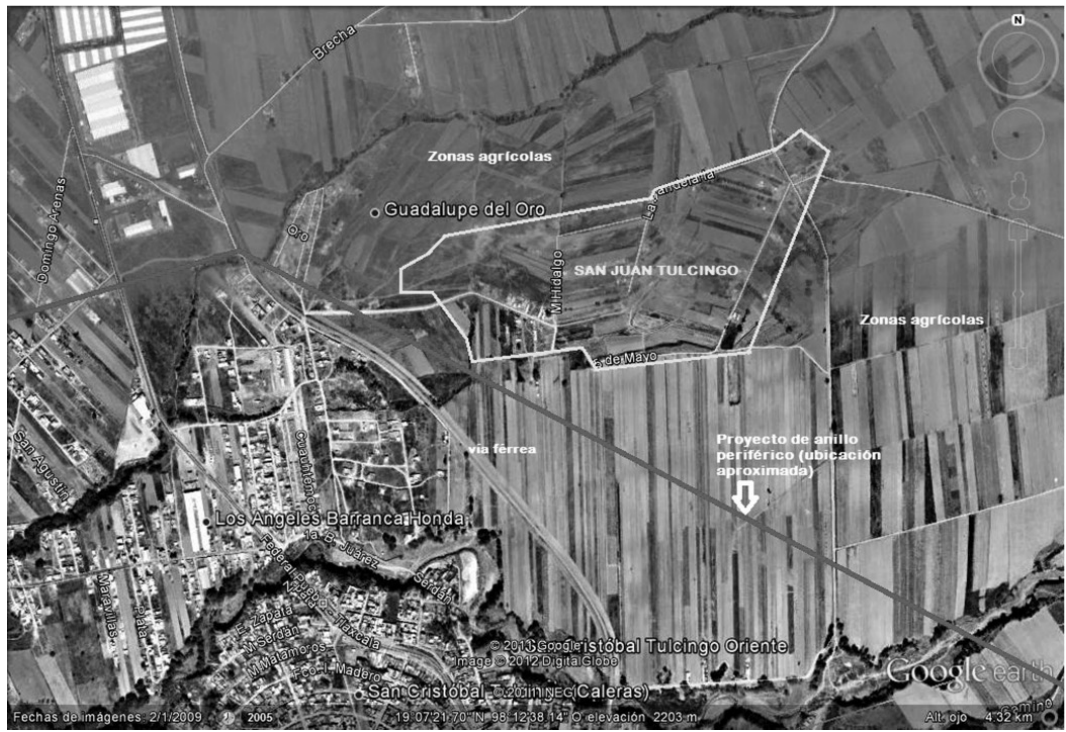

Fuente: Elaboración propia. La imagen fue tomada de Google Earth (2011) y la ubicación aproximada del proyecto del anillo periférico del H. Ayuntamiento del municipio de Puebla (2006: tomo II, p. 12).

El asentamiento de sjt forma parte de las nuevas áreas de crecimiento de la ZMCP y presenta las mismas características de marginalidad urbana de la mayoría de los asentamientos irregulares (AI), es decir, no cuenta con la suficiente infraestructura ni con los equipamientos necesarios para su desarrollo urbano. De hecho, en el asentamiento, la única infraestructura urbana existente es la red eléctrica y en este momento difícilmente podrá acceder a los programas de incorporación al desarrollo urbano ${ }^{6}$ debido a que no cuenta con 50\% de la densidad, como lo marca el artículo 115 de la Ley de Desarrollo Urbano Sustentable del Estado de Puebla (LDUSEP, 2003). Esta situación excluye a los asentamientos que no cumplen con este requisito y es contradictoria a la misma ley que promueve el desarrollo urbano sustentable (título noveno, capítulo III). Además, a pesar de la gravedad urbana ocasionada por los AI, en Puebla y, aunque es creciente el estudio sobre estos asentamientos -Patiño, 2004; Flores, 2004; Reyes,

\footnotetext{
${ }^{5}$ Observamos que las colonias que lo rodean del lado sur-poniente están en proceso de consolidación y al oriente básicamente son zonas agrícolas.

${ }^{6}$ El título noveno, del suelo, capítulo III de la Ley de Desarrollo Urbano Sustentable del Estado de Puebla (2003), señala que los programas de incorporación de los asentamientos humanos al desarrollo urbano son considerados como programas de mejoramiento, a través de los cuales se dota de infraestructura y equipamiento urbanos a los asentamientos irregulares.
} 
1999; López, 1999; Melé, 1994-, todavía resta mucho trabajo en esta materia.

Por otro lado, internacionalmente se desarrollan propuestas urbanas como los ecobarrios (basadas en los principios del desarrollo sustentable) cuya escala urbana de barrio facilita las relaciones entre los diferentes actores. Así, a través de las Agendas 21 locales, los ecobarrios -también conocidos como barrios sustentables- permiten reconstruir las ciudades principalmente en zonas degradadas (industriales y marginales) y prever las nuevas zonas de crecimiento o en proceso de urbanización, con el afán de anticiparse al crecimiento suburbano difuso (Lefèvre y Sabard, 2009), es decir, se utilizan como instrumentos correctivos y preventivos en la planeación.

En los ecobarrios los principios medioambientales adquieren mayor importancia por lo que una de sus características más relevantes es estimular el bajo consumo energético en las viviendas y en la infraestructura urbana, así como promover los espacios verdes en los sitios públicos. No obstante, el aumento de los espacios públicos para fomentar la cohesión social, promover el uso mixto de suelo, y la participación social en el desarrollo de los proyectos, entre lo más destacado, también forman parte de la idea de un ecobarrio (Lefèvre y Sabard, 2009).

Desde los ańos ochenta, principalmente en los países europeos y norteamericanos, se desarrollan este tipo de proyectos de manera creciente. En 2008 Souami (2009) identificó 43 proyectos de ecobarrio en diversos países europeos: Suecia, Alemania, Holanda, Italia, Suiza, España, Inglaterra, Dinamarca, Austria y Finlandia, y 57 en Francia, país en el que en 2006, el gobierno estableció el mandato de construir un ecobarrio antes del 2012 en todas las comunas que tuvieran programas de desarrollo del hábitat significativo (Gobierno de Francia, 2011); el éxito del mandato se intensificó en el 2009 al recibir 160 candidaturas de proyectos de ecobarrio. ${ }^{7}$ En el estudio sobre el potencial de los ecobarrios que hizo Barton (2000) se analizaron 55 de estos, ubicados en diferentes partes del mundo, la mayoría en Estados Unidos de Norteamérica y Europa, pero también en Australia, Rusia, Nueva Zelanda, India, Canadá e Islandia. Los ecobarrios ya empiezan a tener influencia en países latinoamericanos como México, Brasil, Colombia y Chile, entre los más destacados. En el caso de México algunos proyectos recientes giran en torno a los principios de los ecobarrios o barrios sustentables pero muy pocos cumplen con este adjetivo.

\footnotetext{
${ }^{7}$ Para mayor información véase http://www.developpement-durable.gouv.fr/Le-Club-NationalEcoQuartier.html
} 
En esta vorágine internacional de realizar ecobarrios, a veces se dejan de observar las cualidades de sustentabilidad intrínsecas en los asentamientos irregulares; cuando pensamos en este tipo de asentamientos los relacionamos con marginalidad, pobreza y contaminación; sin embargo, también se presentan algunos elementos de cohesión social ${ }^{8}$ como la organización vecinal y el rescate de las tradiciones y costumbres, así como ciertos hábitos, entre ellos, la movilidad activa y la agricultura que, en general, no son considerados en los procesos de mejoramiento urbano oficiales y, lamentablemente, algunas de estas tienden a desaparecer poco a poco ante los procesos de urbanización convencionales.

En este contexto nos planteamos el objetivo de analizar y mostrar las actuales condiciones naturales, urbanas y sociales de San Juan Tulcingo, Puebla, con el afán de reflexionar sobre la posibilidad de desarrollar la idea de un proyecto de ecobarrio en este asentamiento que pueda servir como referente a asentamientos con características similares.

\section{Metodología}

Para acercarnos a nuestro objeto de estudio ha sido necesario un intenso trabajo de campo y de revisión documental en bibliotecas, internet y algunos servicios públicos de Puebla y Cuautlancingo. Consultamos fuentes bibliográficas, nacionales e internacionales, enfocadas en el estudio de los asentamientos irregulares, el desarrollo sustentable y los ecobarrios. Para el entendimiento de las características de los ecobarrios nos basamos en el análisis de la gestión urbana para el desarrollo de ecobarrios en Lille, Francia ${ }^{9}$. También analizamos algunos documentos legales entre los que se encuentran el Programa Municipal de Desarrollo Urbano Sustentable de Puebla (2006), el Programa Municipal de Desarrollo Sustentable de Cuautlancingo (2008) y la Ley de Desarrollo Urbano Sustentable de Estado de Puebla (2003). Otras fuentes documentales locales para hacer el diagnóstico de la parte física-natural y urbana fueron los estudios del Instituto Nacional de Estadística Geografía e Historia (INEGI) y del Instituto de Catastro del Estado de Puebla. Realizamos diversas vistas de campo a San Juan Tulcingo, las cuales nos permitieron ratificar alguna información oficial así como sus rasgos auténticos a través de la observación directa y de entrevistas semidirigidas. Con el trabajo de campo pu-

\footnotetext{
${ }^{8}$ Diversos autores que han realizado estudios sobre asentamientos irregulares ponen en evidencia estas características, por ejemplo: Ramírez, 1993; Cruz, 2001; Bazant, 2004; Patińo, 2004; Bonilla, 2009, entre otros.

${ }^{9}$ Realizada en el Laboratorio de Territorio, Ciudades, Medioambiente y Sociedad TVEs (por sus siglas en francés) del Instituto de Urbanismo de la Universidad de Ciencias y Tecnologías de Lille 1, con una beca posdoctoral del Programa Internacional Erasmus Mundus Lote 20 coordinado por la Dirección de Relaciones Internacionales de la BuAp y la Universidad de Groningen, Países Bajos.
} 
dimos realizar el análisis diagnóstico del lugar y con las entrevistas detectamos las necesidades primordiales, en términos urbanos, de los habitantes y el grado de aceptación de las propuestas, particularmente de la ciclovía, el biodigestor, el huerto urbano y la ampliación de banquetas.

Además realizamos tres sesiones informativas para mostrar los avances de nuestro proyecto y para sensibilizar a los vecinos sobre la importancia del cuidado del medio ambiente, asimismo les mostramos algunas tecnologías alternativas que pueden sustituir las formas convencionales de infraestructura urbana. Hablamos, pues, de un trabajo basado en la investigación-acción que nos ha permitido un aprendizaje recíproco con los habitantes del lugar.

\section{Desarrollo urbano sustentable $v s$ asentamientos irregulares}

Después de la definición del desarrollo sustentable (DS) del informe Brundtland en 1987, y la Conferencia de Río, en 1992, se ha vuelto común hablar de ciudades sustentables, que se caracterizan por buscar su desarrollo en el seno de entornos edificados, armónicos y equilibrados, mediante "la reconfiguración de suburbios desparramados en comunidades de auténticos barrios y diversos distritos, la conservación de los entornos naturales y la defensa del patrimonio arquitectónico" (Ruano, 2000: 19). El desarrollo urbano sustentable, entendido como aquel que se pronuncia a favor de promover ciudades más compactas (caracterizadas por altas densidades de construcción) y complejas, representa un gran desafío sobre todo para los países en vías de desarrollo, en el sentido de que estos países enfrentan serios problemas de control de la urbanización principalmente en la periferia, que es ocupada ilegalmente, en especial, por grupos sociales pobres que forman los llamados asentamientos irregulares.

Los AI no respetan, en general, los lineamientos urbanos, por ejemplo: no dejan superficie para espacios verdes públicos, algunas veces se ubican en zonas de riesgo, es decir, al borde de los ríos, sobre zonas de alta tensión o gaseoductos, lo que los hace vulnerables; además, no cuentan con la infraestructura y servicios básicos, o los tienen marginalmente, entre ellos: agua, luz, drenaje, alcantarillado, servicios viales definidos y urbanizados; en ocasiones son llamados irregulares porque no tienen títulos de propiedad. Las condiciones de urbanización de estos entornos urbanos nos alejan del anhelado desarrollo urbano sustentable. Además, de acuerdo con Fondo Verde (2009a: 5) "el riesgo ambiental de estos establecimientos humanos informales perpetúa el ciclo de la degradación ambiental y contribuye a una mayor vulnerabilidad económica y ambiental, ambos para familias de bajos ingresos y la totalidad del área urbana”. 
Lo anterior se contrapone con algunas de las características del desarrollo urbano sustentable donde la planeación prevé, entre otras cosas: densificación urbana y mezcla de usos de suelo (vivienda, trabajo, equipamientos, recreación), espacios que deben ser acondicionados para favorecer el desplazamiento peatonal (walkable city); incremento de rutas verdes (greenways); espacios agradables y accesibles, tanto para realizar los trayectos como para permanecer en ellos (livable city) (Flores, 2010). Visto de esta manera parecería que los asentamientos irregulares representan el lado opuesto del desarrollo sustentable, no obstante en ellos existen formas no planeadas donde, como lo indican los estudios de Bazant (2001 y 2004), la mezcla de usos de suelo es común; además, existen elementos culturales y de cohesión social fuertes, por ejemplo: la interacción social es estrecha a nivel de manzana, y las fiestas religiosas los llevan a organizarse y mantener las relaciones sociales. Estos últimos elementos son fundamentales en términos de desarrollo sustentable ya que, según Fondo Verde (2009b) permiten trazar planes, programas y estrategias de desarrollo de las regiones y localidades, no sólo considerando las formas de uso de suelo, manejo de recursos y protección del medio ambiente, sino también, como elemento prioritario, aprovechando los conocimientos y riqueza cultural del lugar. Visto de esta manera, los asentamientos irregulares como San Juan Tulcingo pueden alinearse a los principios del desarrollo sustentable como veremos más adelante.

\section{El ecobarrio orígenes y características}

Según Souami (2009), los antecedentes del ecobarrio se remontan a los años ochenta cuando en Austria, los Países Bajos y Alemania aparecen los primeros eco-proyectos situados en las zonas rurales, cuyos creadores le daban una importancia primordial a los aspectos ecológicos urbano-arquitectónicos. Estos proyectos eran elaborados bajo una organización comunitaria. En los noventa, aprovechando eventos de carácter internacional como las exposiciones universales, empiezan a desarrollarse "ecobarrios muestra" al interior de las ciudades (por ejemplo Malmö, Hanovre, París). Se plantean como demostraciones para los técnicos en materia de tecnologías alternativas (celdas fotovoltaicas, reciclaje de aguas pluviales, entre otras) por lo que son considerados como lugares de aprendizaje.

A mediados de la década de los noventa y hasta nuestros días los ecobarrios mantienen un fuerte desarrollo de los elementos medioambientales (sobre todo en lo que se refiere al uso de tecnologías alternativas); sin embargo, han incorporado cada vez más los elementos sociales y económicos del desarrollo sustentable y la participación social como elemento fundamental en la toma de decisiones de este tipo de proyectos. 
Aunque cada ecobarrio tiene sus particularidades podemos identificar en ellos algunas características generales que mencionamos a continuación:

De acuerdo con Barton (2000) un ecobarrio deberá ser tratado como un ecosistema en el sentido de que éste proveerá los elementos esenciales para mejorar la calidad de vida de sus habitantes, creando su propio microclima y las condiciones de confort y sustento en favor de su autonomía. Esto se traduce urbanística y arquitectónicamente en el aprovechamiento y gestión de la energía y del agua, en la disminución del gasto energético y en el aprovechamiento responsable de los recursos naturales. Para ello es necesario el desarrollo y uso de tecnologías alternativas en los edificios y en las infraestructuras urbanas.

La construcción de un ecobarrio, en términos ambientales, significa un trabajo de construcción de la identidad del barrio y de la profundización del sentimiento de pertenencia de sus habitantes (Souami, 2009), así como del aumento de la masa verde y la integración de los espacios naturales con el medio construido.

La movilidad sustentable es una de las características más relevantes de los ecobarrios que, como lo señalan Lefèvre y Sabard (2009), requiere de una reorganización del tejido urbano para reducir los desplazamientos y favorecer otras formas de circulación como las ciclovías y las sendas peatonales (movilidad activa), así como fomentar el uso del transporte público.

Un ecobarrio requiere de una densificación generada por nuevas formas urbanas que rompan con la dispersión pero que no reproduzcan los grandes bloques de vivienda funcionalistas de más de diez niveles. De acuerdo con Barton (2000) se promueve la densificación más alta cerca de las zonas de servicios y, más baja, en las zonas habitacionales con el afán de favorecer un ambiente a la escala humana. La densificación también contribuye a la disminución de los desplazamientos y favorece la proximidad de los servicios.

En un ecobarrio se fomenta la cohesión social a través del aumento de los espacios públicos y de la promoción de actividades, principalmente de sensibilización medioambiental. Como lo muestra Bovet (2009) en su estudio sobre ecobarrios en Europa, en algunos de ellos se construyen granjas o jardines comunitarios para el cultivo de hortalizas de consumo local; estos espacios están acondicionados para realizar talleres de cómo cultivar la tierra y de educación ambiental que permiten, además del aprendizaje, las interrelaciones personales. Otro elemento que se procura integrar en estos proyectos es la mixidad intergeneracional lo cual favorece la solidaridad y estrecha los lazos sociales. Finalmente, las características se presentan en un ecobarrio son la concertación y negociación entre los actores públicos, privados y sociales, mismas que se ha vuelto más ricas y 
complejas. Las sesiones formales e informales para discutir los proyectos son un elemento clave para su elaboración, lo que conlleva, por un lado a mayores tensiones y alargamiento de los procesos para el desarrollo de los proyectos y, por el otro, a promover más la democracia participativa en un marco de gobernabilidad urbana. Para ello se requiere un marco normativo que facilite la implementación de un proyecto con las características de un ecobarrio y que defina los mecanismos de participación social (Lefèvre y Sabard, 2009).

\subsection{Algunos ejemplos de ecobarrios}

Entre los ejemplos más conocidos de ecobarrio a nivel internacional ${ }^{10}$ podemos encontrar: el proyecto de Ecolonia en Alphen aan den Rijn, Holanda, 1993, que se caracteriza por ser una urbanización sensible al medioambiente con la implementación de tecnologías para ahorrar energía, reciclar materiales, aprovechar el agua de lluvia, dar prioridad al peatón, entre otras cosas (Barton, 2000), en este proyecto también se realizaron talleres de arquitectura donde se trabajó de cerca con los habitantes en la elaboración de proyectos de vivienda. El proyecto de Barrio sostenible en Vauban, en la ciudad de Freiburg, Alemania, 1994, antiguo cuartel francés donde las viviendas se construyeron bajo criterios de bajo consumo energético (y en algunos casos con producción de energía); ofrece prioridad al peatón y fomenta el uso del transporte colectivo, maximiza áreas verdes e incentiva el comercio de barrio (uso mixto de suelo), además se realizaron talleres de participación con los vecinos del lugar durante la elaboración del proyecto (Home and Communities Agency, 2010). Este proyecto se encuentra actualmente en su etapa final de construcción. En Francia encontramos el gran proyecto de ecobarrio La Unión, en Lille, cuya realización se prevé en un plazo de 15 años (2006 al 2021), y se caracteriza por integrar elementos tecnológicos con la certificación de Alta Calidad Medioambiental HQE (por sus siglas en francés), mixidad y diversidad social, usos de suelo mixto, empleo local, $30 \%$ de vivienda social, densificación, movilidad sustentable, equipamientos, servicios y espacios públicos accesibles; todo esto en un marco de gestión participativa para lo cual se han creado espacios de diálogo y se han desarrollado diversas dinámicas de participación y consultas públicas (L'Union, 2011).

En el caso de México es conocido el proyecto de ecobarrio (propuesto en 2008) en la colonia Ampliación Michoacana (zona popular del

\footnotetext{
${ }^{10}$ Así, nuestro objetivo al compararlos con México es en el sentido de visualizar la integralidad con la que abordan sus proyectos en términos de desarrollo sustentable, mismos que se deberán aplicar y desarrollar en función de las características sociales, políticas y culturales de cada país.
} 
centro histórico de la ciudad de México) donde se planteó el enverdecimiento del barrio, es decir, se aumentó la superficie verde en los espacios públicos, así también se revistieron bardas con enredaderas, se instalaron baños secos y huertos en las escuelas, alumbrado público con celdas fotovoltaicas para su funcionamiento y se captó el agua de lluvia (Ciudadanos en red, 2008). Este proyecto se limitó a fomentar algunos elementos medioambientales sin tratar de integrar las demás características de los ecobarrios. La realización de este proyecto estaba prevista en un plazo de seis meses. ${ }^{11}$

Aunque no está enfocado en los ámbitos urbanos se menciona el programa de Ciudades Rurales Sustentables -surgido en Chiapas- ya que, en teoría, es lo más cercano a la idea de un ecobarrio. Este programa gubernamental (aplicado en Nuevo Juan de Grijalva, Santiago el Pinar, Jaltenango, Ixhuatán y Emiliano Zapata) tiene como objetivo adecuar la distribución territorial de la población y reubicar a las poblaciones que viven dispersas y lejos de servicios básicos como clínicas, escuelas y templos religiosos, esto en el marco del desarrollo sustentable. El primer proyecto, realizado en casi dos años, ${ }^{12}$ fue Nuevo Juan de Grijalva. Urbanísticamente cuenta con utilización de celdas solares para el alumbrado público y biodigestores para el tratamiento de aguas pluviales; vialidades vehiculares y peatonales, jardines vecinales y canchas de usos múltiples. Las viviendas (de adoblock y láminas especiales), cuentan con traspatios productivos para el cultivo de hortalizas, frutos y crianza de animales para el autoconsumo (Gobierno del Estado de Chiapas, 2011). Aunque la idea de Chiapas contiene claramente en su discurso los principios generales de la sustentabilidad ha sido fuertemente criticada por expertos, ya que como se comentó en el Primer Encuentro sobre Ciudades Rurales Sustentables: Las experiencias y los proyectos, ${ }^{13}$ los modelos de vivienda -con tecnologías holandesas- no fueron concebidos en función de la idiosincrasia y costumbres de la gente que fue reubicada en estos sitios, de manera que muchos han regresado a sus territorios originales y algunas viviendas se convirtieron en cantinas y lugares donde se ejerce la prostitución. De acuerdo con los especialistas, una de las causas principales del fracaso de Chiapas es no haber realizado el proyecto con los habitantes (Hernández, 2011), es decir, no hubo una gestión participativa.

${ }^{11}$ En el proyecto de ecobarrio Ampliación Michoacana (en la Delegación Venustiano Carranza) el jefe delegacional anunció en julio de 2008 que la "eco-munidad” (no le llamó ecobarrio) estaría lista antes de diciembre (se sobrentiende que del mismo año). Cabe aclarar que se refería a acciones como la instalación de baños secos en las escuelas y plantas de tratamiento de aguas grises (Conavi, 2008:19).

${ }^{12}$ La premura del tiempo de realización se debió a la necesidad de albergar a los habitantes que habían sufrido el desgajamiento de un cerro sobre el río Grijalva, es decir, 410 familias damnificadas.

${ }^{13}$ Este encuentro fue realizado en la ciudad de Puebla debido a que el gobernador de este estado, Moreno Valle, quiso replicar la experiencia chiapaneca. 
Cabe mencionar que aunque en México las acciones gubernamentales son incipientes en materia de ecobarrios, encontramos proyectos, generalmente de organizaciones y cooperativas ciudadanas, que promueven la permacultura, las granjas familiares y el desarrollo comunitario en armonía con la naturaleza. Por ejemplo, en el Centro de Agroecología y Vida Sostenible, administrado por la Cooperativa las Cańadas ${ }^{14}$ en Huatusco, Veracruz, se promueve el trabajo cooperativo para producir alimentos como maíz, frijol, tubérculos, hortalizas, huevos y leche para el autoconsumo, así como la utilización de algunas tecnologías no contaminantes como los sanitarios ecológicos. Las organizaciones ciudadanas también ayudan a capacitar y sensibilizar a las personas principalmente en materia medioambiental y de cooperación, así como en la participación ciudadana. ${ }^{15}$

Como podemos observar, en los casos europeos el desarrollo de los ecobarrios se ha planteado desde un enfoque integral donde el trabajo conjunto con los habitantes es fundamental para guiar y diseñar el proyecto en función de sus necesidades; esto, según Souami (2009), influye en que el tiempo promedio para la construcción de los ecobarrios sea de 10 años. En los casos mexicanos los plazos de realización de proyecto son de corto plazo, hecho que dificulta una participación social efectiva. Además, están muy enfocados a trabajos técnicos, como en el caso de la Ampliación Michoacana y, en el caso de las localidades rurales sustentables no alcanzan a realizar un proyecto integral de los principios del desarrollo sustentable.

\section{El doble rostro de San Juan Tulcingo}

A través del estudio de San Juan Tulcingo pudimos descubrir su doble rostro: por un lado, el que nos muestra algunas características del desarrollo sustentable y, por el otro, el de la precariedad de las condiciones físico-urbanas y económicas de los habitantes.

Respecto a su primer rostro, encontramos que una de sus características más relevantes es la cohesión social de sus habitantes. Aunque no existen las condiciones urbanas (espacios públicos) en sjt la poca población, así como sus afinidades religiosas (practican el catolicismo) permiten a los habitantes interactuar con mayor frecuencia. La forma más común de abastecerse de productos básicos para el consumo cotidiano es a través de las escasas misceláneas y verdulerías que al mismo tiempo son una

\footnotetext{
${ }^{14}$ Para mayor información consultar http://www.bosquedeniebla.com.mx/

15 Para mayor información consultar: http://ecoaldeas.bligoo.com/content/view/707669/ Ecoaldeas-proyectos-sustentables-y-permacultura-en-Mexico.html; http://www.huehuecoyotl.net/; http://www.reddeecoaldeas.org/rem/news.php
} 
pequeña fuente de ingreso para los propietarios. Otra fuente de ingreso son los oficios, practicados principalmente por los hombres, como la herrería y la carpintería; por su parte las amas de casa realizan manualidades como el tejido, juguetería y arreglos florales artificiales que venden entre los lugareños. La celebración de las fiestas religiosas despierta mucho interés, comunicación y organización entre los habitantes, tal es el caso del Día de Muertos, Semana Santa y la fiesta de su patrono: San Juan, el 24 de junio. Esta última reúne a los vecinos en el terreno donde está su capilla; lonas, unas cuantas mesas, música y cohetes son el escenario de una gran fiesta. Una escena cotidiana son las pláticas entre vecinas por las tardes (una vez que se han liberado de los quehaceres del hogar) quienes se reúnen en la avenida principal y al mismo tiempo vigilan a sus niños que juegan en las calles de terracería.

Por otro lado, las caminatas y la bicicleta son las formas más comunes de desplazamiento practicadas al interior del asentamiento. Ningún transporte público circula en la zona (la mayoría no tiene automóvil), por lo tanto tienen que ir caminando hasta la carretera federal Puebla-Tlaxcala, localizada a veinte minutos aproximadamente del asentamiento. El hecho de que todas las calles sean de terracería, provoca polvaredas y en tiempos de lluvia los lodazales dificultan circular en ellas. Ante esta situación, una de las principales demandas de la población es contar con el servicio de transporte público pero que "dejen el espacio para andar en bicicleta y caminar tranquilamente", es decir, quieren preservar las formas de desplazamiento a las que están habituados.

En cuanto a su medio físico natural, la principal riqueza radica en la abundancia de agua, ello gracias a mantos freáticos que son alimentados por los afluentes de la montaña de la Malinche y por la permeabilidad de sus suelos (gleysol, feozem y vertisol). Esta situación permite el abastecimiento regular de agua para las viviendas a través de los pozos, sin embargo, no cuentan con red de agua potable. Su clima templado-húmedo con lluvias en verano y su temperatura media anual de $17.5^{\circ} \mathrm{C}$ acrecientan su riqueza hídrica, principalmente para la agricultura. En las calles y caminos vecinales se encuentran, escasamente, árboles de eucalipto, trueno y casuarina. Desgraciadamente la fauna ha ido perdiéndose conforme avanza la mancha urbana, ocasionalmente se llegan a encontrar liebres, tlacuaches, topos, zopilotes, víboras de cascabel y algunos pájaros.

En sjt existen prácticas de carácter rural que son para el autoconsumo, tal como la agricultura de temporal, la ganadería y la apicultura. Su principal cultivo es el maíz y son los adultos y algunos jóvenes quienes se encargan de trabajar las tierras, no obstante, esta práctica se va perdiendo debido a los cambios de uso de suelo de rural a urbano, situación que preocupa a los habitantes debido a que es una importante fuente de ali- 


\section{Figura II}

Calles de terracería, vivienda marginal y escasa infraestructura son parte de la imagen de San Juan Tulcingo

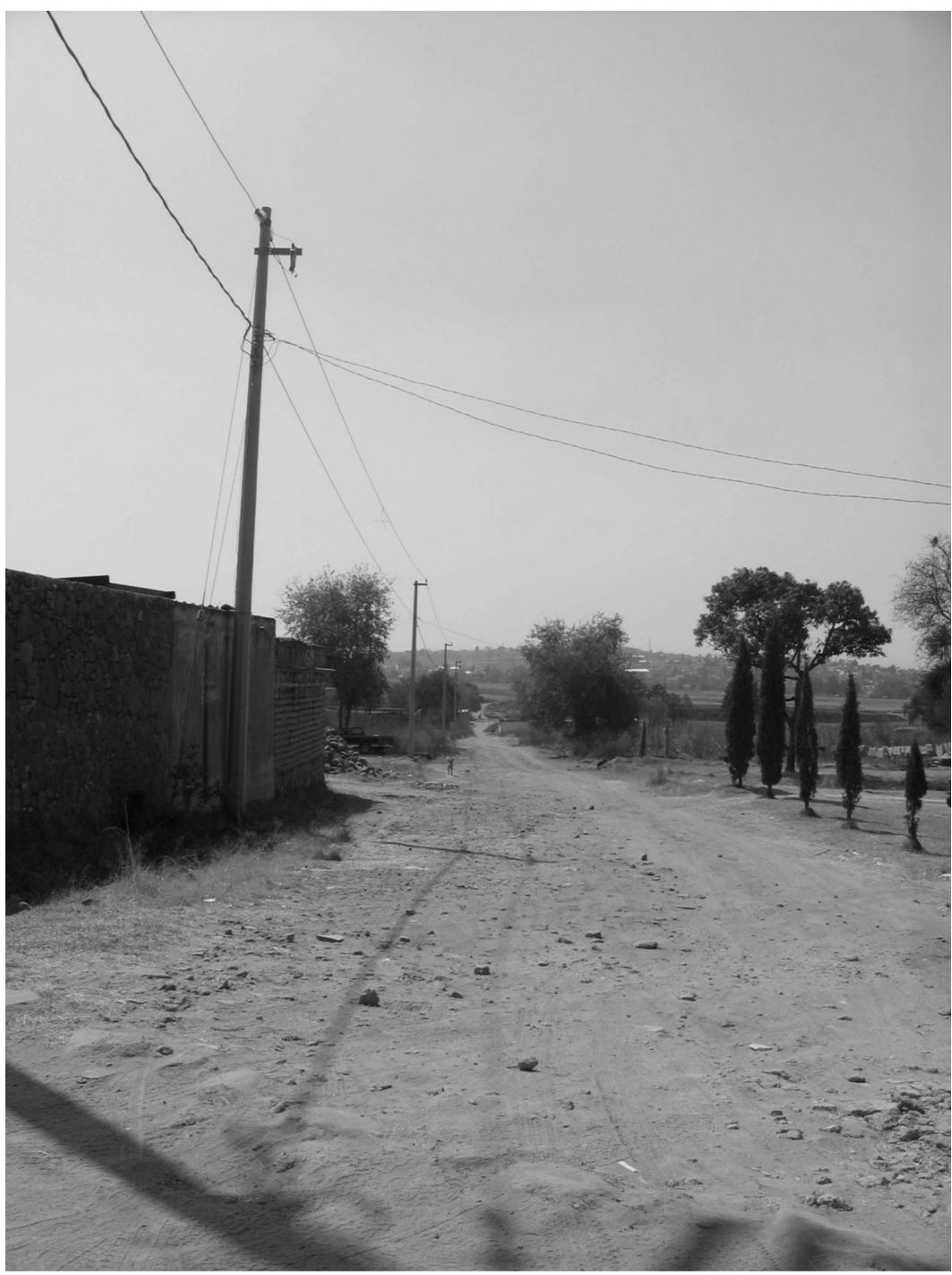

Foto: Lourdes Flores, 2009. 
mentación, además de que en su fiesta patronal, el consumo del maíz -en varias de sus formas culinarias: esquites, chileatole, elotes, entre otras- es parte fundamental de sus costumbres. Respecto a la ganadería se crían ganado bovino, porcino y ovino; estos animales, que son pocos, se cuidan en los patios de las casas y en algunos terrenos baldíos.

En contraparte, el otro rostro de sJT muestra las condiciones físicourbanas y económicas precarias de sus habitantes. La carencia de la infraestructura y servicios básicos es evidente, en realidad sólo cuentan con el servicio de luz en vivienda, alumbrado público y con red telefónica (figura II). El desecho de aguas residuales, jabonosas y negras, en los casos donde no tienen fosa séptica se hace directamente en las calles ocasionando insalubridad en el ambiente (contaminación de suelos y agua), misma que se acentúa con los desechos de basura vertidos directamente en la barranca (ubicada a aproximadamente quince minutos de distancia del asentamiento) o quemándola. Raras veces la tiran directamente sobre los terrenos baldíos.

El único servicio educativo con el que cuentan es un preescolar y, el cual fue adaptado en una casa. Su principal fuente de abastecimiento de alimentos es la central de abastos, localizada a veinticinco minutos en autobús por la carretera federal Puebla-Tlaxcala.

La vivienda es, en general, de autoproducción (de uno o dos niveles), utilizan principalmente el block, ladrillo, lámina y la vigueta bovedilla; se construye por etapas. Sólo hay 60 viviendas, la mayoría no tiene acabados y se ubican en terrenos cuya superficie varía de los $150 \mathrm{~m}^{2}$ (cuando, ya están sublotificados) hasta los $5000 \mathrm{~m}^{2}$ (producto de la lotificación de los terrenos agrícolas). Es común que en la vivienda existan varias habitaciones que se construyen de acuerdo a sus necesidades: "conforme la familia va creciendo".

La carencia de espacios públicos y lugares de entretenimiento ocasiona que los niños jueguen en las calles y los jóvenes busquen en el centro de la ciudad de Puebla y en los centros comerciales espacios para su esparcimiento; pocos pasean en la zona.

\subsection{Las acciones de las autoridades locales en San Juan Tulcingo}

Debido a que existe un conflicto de definición de límites territoriales en SJT, las autoridades del municipio de Puebla y las de Cuatlancingo (quienes se disputan el territorio) no se han puesto de acuerdo para atender las necesidades de este asentamiento. Oficialmente el uso de suelo en esta zona para el municipio de Cuautlancingo permanece como agrícola-urbano y para el municipio de Puebla, como agrícola; sin embargo, las autoridades de Puebla les otorgaron el servicio de energía eléctrica. Res- 
pecto al resto de los servicios, las autoridades locales han hecho caso omiso a las peticiones de los habitantes; mencionan los vecinos que "las autoridades sólo se acercan en tiempos electorales, nos hacen muchas promesas y luego nunca regresan".

Hasta ahora, son las autoridades de Cuautlancingo con quienes tienen mayor contacto pero no les han reconocido el cambio de uso de suelo y, por lo tanto, no cuentan con alineamiento y número oficial; sin embargo, en este municipio es donde pagan el predial.

Ante la indiferencia de las autoridades los vecinos de San Juan Tulcingo se han organizado para adherirse a la organización de colonos Dignificación Derechos Humanos y Desarrollo Social, con el afán de presionar a las autoridades locales para que les doten de los servicios públicos y los incorporen al desarrollo urbano de la Zona Metropolitana de la Ciudad de Puebla. Esta organización, creada en el 2009 como asociación civil, tiene como objetivo fundamental impulsar el desarrollo de las colonias adheridas (todas las colonias se ubican al norte de la ciudad de Puebla), a través de la creación de espacios para el desarrollo social y capacitación. Son intermediarios entre colonos y autoridades para plantear propuestas de mejoramiento en sus zonas. Gracias a esta asociación los vecinos han logrado organizarse para realizar sus demandas de una manera ordenada e interactuar con vecinos de otros asentamientos que se encuentran en condiciones similares y ayudarse mutuamente, hecho que ha reforzado la cohesión social en el asentamiento. No obstante, sus esfuerzos hasta ahora han sido nulos, ya que las autoridades municipales, basándose en el artículo 115 sobre la ocupación mínima que deben tener los asentamientos para acceder a los programas de incorporación al desarrollo urbano que mencionamos en la introducción, no plantean ningún tipo de mejoramiento urbano en sJT. Ante esta situación resulta fundamental que las autoridades pongan atención al principio de prevención ${ }^{16}$ que plantea la necesidad de realizar todas las acciones previas orientadas a evitar conductas agresivas sobre el medio natural; y que desarrollen acciones para (como lo marca el artículo 113 párrafos I y II de la LDUSEP, 2003) "reordenar áreas de los centros de población deterioradas física o funcionalmente" y "coadyuvar en el crecimiento ordenado de los centros de población".

El estado de invisibilidad de sjt ante las autoridades locales y la indefinición de las competencias administrativas, para saber a cuál de los dos municipios se deben dirigir los vecinos al realizar sus gestiones, dificulta

${ }^{16}$ El Principio de Prevención se encuentra en varios documentos, por ejemplo, en la Declaración de Principios sobre la Lucha contra la Contaminación del Aire (1968), en la Carta de los Suelos (1972), en la Declaración de la Conferencia Internacional de Tbilisi (1977), en la Declaración de Nairobi (1982) y en los Programas de Acción Comunitaria en Materia de Ambiente de la ue (el más reciente es de 2010). 
y complica su proceso de crecimiento y consolidación urbana dirigiéndolo hacia la precariedad urbana, característica general de los asentamientos irregulares.

\subsection{La participación social en el desarrollo del proyecto}

La participación de los habitantes para el desarrollo de nuestro proyecto de ecobarrio ha sido un elemento clave para elaborar el diagnóstico y empezar a plantear algunas estrategias de diseño urbano y establecer las prioridades del proyecto. Hasta ahora hemos tenido tres encuentros formales y varios informales (figura III) con los vecinos de la colonia para conocer sus principales preocupaciones y necesidades, y para darles información sobre las ventajas de crear espacios públicos, aumentar áreas verdes, proponer diferentes medios de desplazamiento, reciclar la basura, entre otros. Sin embargo, podríamos calificar su participación de tímida, ya que no han tenido una postura crítica que nos haga cambiar la dirección de nuestra propuesta. Nos externaron su completa confianza y aprobación para el desarrollo del proyecto. De acuerdo con los diálogos que hemos tenido se conforman con obtener el trabajo técnico para el sistema de drenaje. En contraste con lo anterior, la relación entre ellos para decidir sus acciones es muy fuerte, hecho que observamos principalmente al final de las reuniones, cuando empezaban a discutir cómo organizarse para gestionar nuestra propuesta ante las autoridades locales.

La pequeña escala ha permitido entablar relaciones muy directas con los vecinos, entender sus preocupaciones y necesidades. A partir de este contacto también podemos conocer la aceptación para el desarrollo de un proyecto con características de sustentabilidad urbana. Por ejemplo, $75 \%$ de la población está de acuerdo en hacer una ciclovía, ya que es un medio que utilizan para desplazarse al interior de la colonia e incluso para ir a las colonias vecinas. Mientras $85 \%$ aprueba la construcción de banquetas amplias, $84 \%$ acepta la idea de crear espacios comunales para el cultivo de hortalizas, y así, 100\% de los consultados están dispuestos a realizar prácticas de reciclaje. En materia medioambiental existe preocupación entre los vecinos por la pérdida de vegetación y fauna del lugar, así como por la contaminación producida principalmente por las descargas de agua residual y la basura. Los vecinos mostraron su interés y disposición para impulsar el desarrollo de tecnologías alternativas en las infraestructuras, particularmente para el sistema de drenaje donde planteamos un proyecto detallado para la construcción de un biodigestor. Respecto a este proyecto, gracias a la organización vecinal, ya se logró obtener la donación de un terreno para construir el biodigestor, esto a través de la negociación directa entre ellos. 


\section{Figura III}

\section{Presentación de los avances del proyecto de ecobarrio ante los vecinos de San Juan Tulcingo}

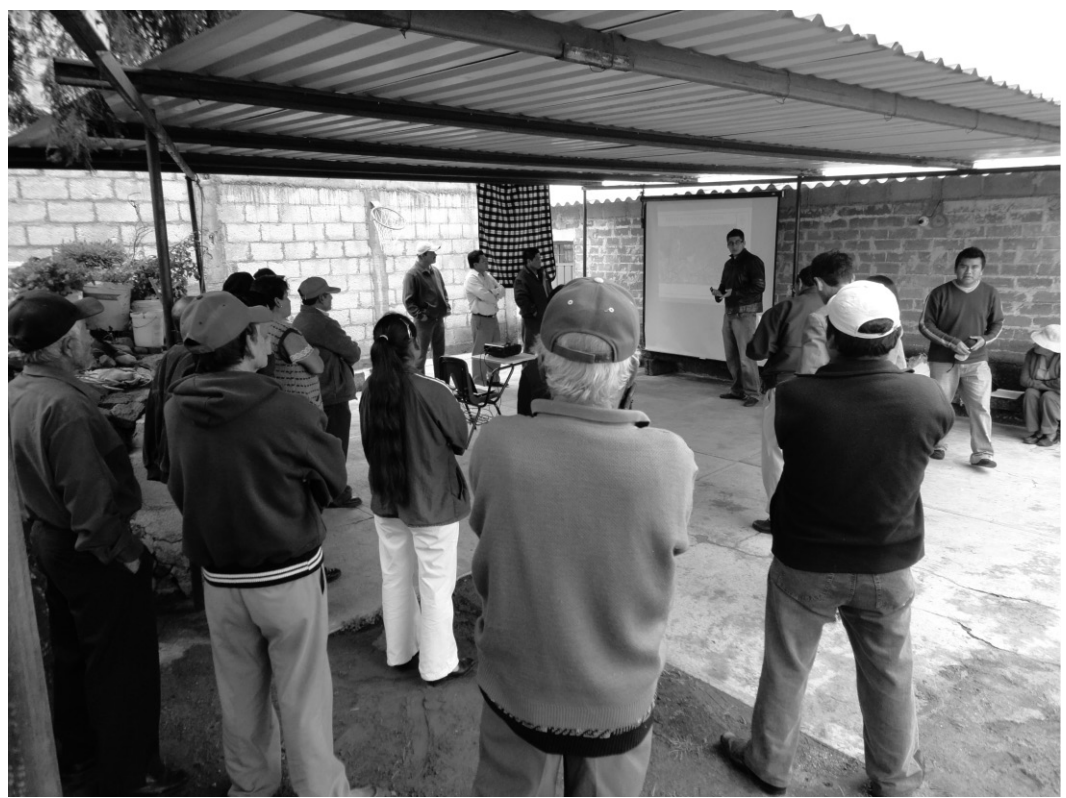

Foto: Lourdes Flores, 2010.

Así, una de las potencialidades más destacadas que hemos identificados en San Juan Tulcingo es su capacidad de organización y cohesión social para mejorar sus condiciones actuales de entorno de vida, elemento fundamental de los procesos de gobernabilidad con participación social.

\section{Conclusiones}

En las últimas dos décadas los ecobarrios aparecen, principalmente en los países desarrollados, como una forma alternativa para redirigir y mejorar los barrios en las zonas urbanas, así como para prever el crecimiento ordenado y sustentable de las ciudades. El abordaje integral de los elementos medioambientales, sociales y económicos es cada día más complejo, sin embargo, la escala de barrio permite un mejor manejo de ellos y sobre todo la interacción y negociación entre los diferentes actores involucrados.

No se trata de replicar proyectos extranjeros, sino de observar la integralidad y particularización con la que son abordados. Como lo menciona Tudela (2001: 44) "la opinión pública necesita entender que la tecnología es una variable, no un parámetro constante administrado por una secta especializada; tendrá que asimilar la existencia de opciones 
diferentes, y comprender las principales implicaciones sociales y ambientales de cada una de ellas", lo que conlleva a un análisis específico de las condiciones de contexto donde se quiera desarrollar un proyecto de este tipo, y un trabajo conjunto y articulado entre los diferentes actores públicos, privados y sociales. De lo contrario, tendremos experiencias fallidas de proyectos como Juan de Grijalva en Chiapas, o proyectos como el de la colonia Ampliación Michoacana en la ciudad de México y que son llamados, en este último caso, ecobarrios pero que sólo atienden técnicamente una de las variables del desarrollo sustentable: el medioambiente.

Para el caso de los asentamientos irregulares los ecobarrios podrían representar una alternativa para controlar el crecimiento marginal periférico por su carácter correctivo y preventivo. En el caso de San Juan Tulcingo, su incipiente urbanización representa un momento oportuno para desarrollar un proyecto de ecobarrio que redirija su actual tendencia de urbanización marginal. Consideramos que, tomando en cuenta los elementos de sustentabilidad que encontramos en sjt y los principios para el desarrollo de los ecobarrios, podríamos tener una fórmula alternativa que complementaría los programas de incorporación al desarrollo urbano de los asentamientos irregulares que actualmente desarrolla el gobierno local. Este planteamiento lo hacemos a nivel experimental considerando las experiencias ya realizadas en otros lugares y, por supuesto, tomando como base las condiciones de contexto en Puebla.

Estamos conscientes de que una propuesta de este tipo demandaría, además de una fuerte voluntad política, la generación de mecanismos de colaboración y participación entre los actores involucrados, más talleres de sensibilización ambiental (donde las asociaciones serían de gran ayuda) y un conocimiento mayor de los elementos teóricos y prácticos de los ecobarrios.

\section{Bibliografía}

Azuela, Antonio (1999), La ciudad, la propiedad privada y el derecho, El Colegio de México, México.

Barton, Hugh (2000), Sustainable communities. The potential for econeighbourhoods, Earthscan, Londres.

Bazant S., Jan (2004), Asentamientos irregulares. Guia de soluciones urbanas, Trillas, México. 
Bazant S., Jan (2001), Periferias urbanas. Expansión urbana incontrolada de bajos ingresos y su impacto en el medio ambiente, Trillas, México.

Bonilla-Rodríguez, Roberto (2009), "Agricultura y tenencia de la tierra en Milpa Alta. Un lugar de identidad", Argumentos, 22 (61), Universidad Autónoma Metropolitana Xochimilco, México, pp. 249-282.

Bovet, Philippe (2009), Écoquartiers en Europe, Terre vivant, Mens, Francia.

Ciudadanos en red (2008), "Plantan ecobarrio en zona urbana", http:// www.metropoli.org.mx/node/4791, 10 de octubre de 2010.

Conavi (Consejo Nacional de la Vivienda) (2008), "Síntesis Informativa”, http://www.conafovi.gob.mx/sintesis/Julio2008/Sintesis_Julio_01_2008.pdf, 28 de octubre de 2010.

Cruz-Rodríguez, Ma. Soledad (2001), Propiedad, poblamiento y periferia rural en la Zona Metropolitana de la Ciudad de México. Universidad Autónoma Metropolitana Azcapotzalco/Red de Investigación Urbana, México.

Flores-Lucero, Lourdes (2010), El desarrollo urbano sustentable en la ciudad de Montreal / Le développement urbain durable dans la ville de Montréal, Fomento Editorial BuAp, Puebla.

Flores-Lucero, Lourdes (2004), "La gestión urbana en la periferia de la ciudad de Puebla, México 1990-2000. Los asentamientos irregulares ubicados en las zonas ejidales de las colonias populares", tesis doctoral, Universidad Politécnica de Cataluña, Barcelona.

Fondo Verde (2009a), "Teoría de la gestión ambiental", en Diplomado en gestión ambiental urbana, módulo 3, Organización de Estados Americanos, Perú, pp.1-20.

Fondo Verde (2009b). “Teoría del desarrollo sustentable", en Diplomado en gestión ambiental urbana, módulo 4, Organización de Estados Americanos, Perú, pp.1-34. 
Gobierno del Estado de Chiapas (2011), "Ciudades Rurales Sustentables", Instituto de Población y Ciudades Rurales. http://www.ciudadesrurales.chiapas.gob.mx/, 15 de septiembre de 2011.

Gobierno del Estado de Puebla (2003), Ley de Desarrollo Urbano Sustentable del Estado de Puebla. Dirección General de asuntos jurídicos, de estudios y proyectos legislativos, Puebla, México.

Gobierno de Francia, Ministerio de Ecología, del Desarrollo Sustentable, Transportes y Vivienda (2011) "La Grenelle de l'environnement", http://www.legrenelle-environnement.fr/, 25 de septiembre de 2011.

H. Ayuntamiento del Municipio de Puebla (2006), programa Municipal de Desarrollo Urbano Sustentable de Puebla, tomo II, Puebla.

Home and Communities Agency (2010), Eco-Towns: Freiburg (Germany), Leading environmental policy and best practice, http://showcase. homesandcommunities.co.uk/case-study/ecotowns-freiburggermany.html\#background, 20 de septiembre de 2010.

Hernández-Alcántara, Martín (2011), "Rechazan académicos y organizaciones modelo de Ciudades Rurales Sustentables para Puebla", La Jornada de Oriente, http://www.lajornadadeoriente.com. mx/2011/09/05/puebla/jus103.php, 5 de septiembre de 2011.

L'Unión (2011), L'écoquartier pilote de la métropole lilloise, http://www. lunion.org/eco-quartier/eco-quartier-de-l-union.html,19 de septiembre 2011.

Lefèvre, Pierre y Michel Sabard (2009), Les écoquartiers, Apogée, Francia.

López-Tamayo, Nicolás (1999), "La urbanización periférica. Mercado de suelo y urbanización en la ciudad de Puebla 1970-1990", tesis de doctorado, unam, México.

Melé, Patrice (1994), Puebla, urbanización y políticas urbanas, Benemérita Universidad Autónoma de Puebla-Universidad Autónoma Metropolitana Azcapotzalco, México.

Patiño-Tovar, Elsa (2004), "Periferia poblana: la desigualdad del crecimiento", Papeles de población, núm. 042, Universidad Autónoma del Estado de México, México, pp. 125-151. 
Ramírez-Saiz, Juan Manuel (1993), La vivienda popular y sus actores, Red Nacional de Investigación Urbana, Guadalajara-Universidad de Guadalajara, Centro de investigaciones sobre movimiento sociales, México.

Reyes-Mendiola, Rosalía (1999), "Integración de las colonias periféricas a la estructura urbana de la ciudad de Puebla", tesis de maestría, Benemérita Universidad Autónoma de Puebla, Puebla.

Ruano, Miguel (2000), Ecourbanismo. Entornos humanos sostenibles: 60 proyectos, Gustavo Gili, Barcelona.

Souami, Taoufik (2009), Écoquartiers. Secrets de fabrication. Analyse critique d'exemples européens, Ediciones Les Carnets de l'info, Paris.

Tudela, Fernando (2001), "El Laberinto de la complejidad. Hacia un enfoque sistémico del medio ambiente y la gestión de los servicios urbanos", en Schteingart y d'Andrea, Servicios urbanos, gestión local y medio ambiente. El Colegio de México-CE.R.FE, México, pp. 41-51.

Recibido: 21 de octubre de 2010. Reenviado: 29 de septiembre de 2011. Aceptado: 15 de enero de 2012.

María de Lourdes Flores-Lucero. Es doctora en gestión y valoración urbana y maestra en valoraciones inmobiliarias, ambos grados por la Universidad Politécnica de Cataluña, Barcelona, España. Actualmente es profesora e investigadora en la FA-BUAP. Es miembro del Sistema Nacional de Investigadores, nivel I. Su línea de investigación es sustentabilidad y desarrollo urbano sustentable, realiza proyectos relacionados con la evaluación de la sustentabilidad en planes y proyectos urbanos y el desarrollo de ecobarrios. Entre sus últimas publicaciones se encuentran: "En busca de la gestión participativa: el caso del proyecto del ecobarrio de la Unión, Lille, Francia”, Revista INVI, Instituto de la Vivienda-Facultad de Arquitectura y Urbanismo-Universidad de Chile, vol. 27, núm. 76, pp. 203-221 (2012); "Una herramienta clave, los ecobarrios", Ciudades, Red Nacional de Investigación Urbana, 91, Puebla, pp. 37-42 (2011); El desarrollo urbano sustentable en la ciudad de Montreal / Le développement urbain durable dans la ville de Montréal, Dirección de Fomento EditorialFA-BUAP, (2010) y "Progress towards sustainability in planning: San 
Francisco and Montreal", en Focus, Journal of the City and Regional Planning Department, California Polytechnic State University San Luis Obispo, vol. 7 (1), pp. 69-75 (2010). Ha realizado estancias de investigación posdoctoral en el Instituto de Urbanismo de la Universidad de Montreal (u de M), Canadá, y actualmente en el Laboratorio de Territorios, Ciudades, Medioambiente y Sociedad TVEs (por sus siglas en francés) de la Universidad de Lille 1, Francia. Además de una estancia corta en el Departamento de Planeación Urbano Regional del Politécnico de California (CALPOLY), EEUU. 